\title{
OBSTRUCTED OBTURATOR HERNIA IN IMMEDIATE POSTOPERATIVE PERIOD: A RARE DIFFERENTIAL DIAGNOSIS
}

Minakshi Gadhireㄹ, Kanchan Sachanandani², Prashant Kadam³ ${ }^{3}$ Mohan Joshi ${ }^{4}$

\section{HOW TO CITE THIS ARTICLE:}

Minakshi Gadhire, Kanchan Sachanandani, Prashant Kadam, Mohan Joshi. "Obstructed Obturator Hernia in Immediate Postoperative Period: A Rare Differential Diagnosis". Journal of Evolution of Medical and Dental Sciences 2014; Vol. 3, Issue 74, December 29; Page: 15600-15602, DOI: 10.14260/jemds/2014/4104

\begin{abstract}
An Obturator hernia is a rare condition but is associated with highest mortality of all abdominal wall hernias. Early surgical intervention is often hindered by clinical and radiological diagnostic difficulty. This case report highlights the obstructed obturator hernia occurring as a postoperative complication in the immediate postoperative period. We present the case of a 65-yearold lady who was operated for open cholecystectomy and developed acute postoperative small intestinal obstruction. Obturator hernias are notorious for diagnostic difficulty. However early CT imaging reduces the morbidity and mortality. A high index of suspicion is required even in immediate postoperative period on $2^{\text {nd }}$ or $3^{\text {rd }}$ postoperative days as an alternative to paralytic ileus in assessing patients with small bowel obstruction. Rapid clinical and appropriate radiological assessment followed by surgery is critical to successful treatment.
\end{abstract}

KEYWORDS: Intestinal Obstruction, Obturator Hernia.

INTRODUCTION: Obturator hernias are infrequent with incidence of $0.7-1 \%$ and account for 0.2 $1.6 \%$ of all cases of mechanical obstruction of small bowel.(1) Highest mortality of all abdominal wall hernias is also attribute to it ranging $13-40 \%$. $^{(2)}$

Obturator hernia sac passes through obturator foramen following the path of obturator nerve and vessels. Signs and symptoms of obturator hernia are nonspecific as there is rarely any palpable mass as in inguinal hernias nor are the legendary signs of Howship-Romberg and Hannington-Kiff positive in all patients and diagnostic imaging also can often be inconclusive. The combination of diagnostic difficulty and high mortality rates make obturator hernia a serious diagnosis that can be potentially be overlooked.

We present an interesting case of a thin elderly female who presented with intestinal obstruction on fourth postoperative day and diagnosed with obstructed obturator hernia on CT imaging.

CASE REPORT: A 65-year-old thin and frail lady with old case of Pulmonary Koch's operated for Open Cholecystectomy for suspected Gall bladder carcinoma. Intraoperatively, Gall bladder was free of any adhesions and Calots triangle was not frozen; also there were no liver or peritoneal metastases. Ryles tube was removed next day. On postoperative day 3, she complained of multiple episodes of bilious vomiting with gradual distension of abdomen and no passage of flatus. Abdominal $\mathrm{X}$-ray revealed dilated small bowel loops with multiple air-fluid levels and gasless colon. Conservative treatment started in view of post-operative paralytic ileus. But considering the progressive nature of obstruction, CECT of abdomen was done which was suggestive of obstructed right-sided obturator hernia (Fig 1) She was immediately explored through midline laparotomy, which revealed a Richter's type Hernia of ileum into a projection of peritoneum through right obturator canal (Fig 2) \& (Fig 3) 
Small bowel wasn't ischemic hence, fortunately no resection was required. There were also two more passable stricturous bands in proximal ileum suggestive of previous episodes of spontaneously resolving sub-acute intestinal obstruction in obturator hernia going unnoticed. The peritoneal out folding was closed primarily with Polypropylene suture and re-enforced with Sepramesh 2"x2" over the defect. Patient had uneventful postoperative recovery (Fig 4) At follow up she was diagnosed with Papillary Adenocarcinoma of Gall bladder but exhibited no abdominal pain or bowel complaints.

DISCUSSION: Arnaud de Ronsil first described Obturator hernia in 1724.(3) With the nickname of 'little old lady's hernia', it usually occurs in multiparous and emaciated women due to wide pelvis and enlarged obturator canal. Risk factors are COPD, Ascites and Chronic constipation. Cardinal clinical symptom is acute intestinal obstruction. Early diagnosis is challenging due to nonspecific signs and symptoms. CT imaging has superior sensitivity and accuracy in the diagnosis.

In our case, preoperative diagnosis was made by CT and Emergency operation was done. Fortunately, bowel was not ischemic and recovery was rapid.

The only treatment of Obturator hernia is Surgery due to high chances of strangulation and high morbidity and mortality. The various approaches are trans-peritoneal, retro-pubic and inguinal. Midline Laparotomy was used in this case for facilitation of bowel resection if necessary. The various methods of repair are simple suture closure, closure of obturator defect with adjacent tissue like bladder, and meshplasty.(4,5,6) In our case, we did simple suture closure with meshplasty and outcome was satisfying.

CONCLUSION: Obturator hernia remains an unusual but important diagnosis to consider in elderly, thin patients with intestinal obstruction. Delay in diagnosis and surgery in addition to advanced age and associated comorbidities contribute to high morbidity and mortality. A high index of suspicion should always be present even in acute postoperative period where it may go unnoticed and land up in unnecessary bowel resection and sepsis.

\section{REFERENCES:}

1. S.K. Mantoo, K. Mak, T.J. Tan. Obturator hernia: diagnosis and treatment in the modern era. Singapore Medical Journal 2009; 50: 865-870.

2. L.De Clerq, K. Coenegrachts, T. Feryn, A. Van Couter, et al. An elderly woman with obstructed obturator hernia: a less common variety of external abdominal hernia. Journal Belge de Radiologie. Belgisch Tidschriftvoor Radiologi 2010; 93: 302-4.

3. Ziegler DW, Rhoads JE Jr. Obturator hernia needs a laparotomy, not a diagnosis. Am J Surg.1995; 170: 67-68.

4. Lo CY, Lorentz TG, Lau PWK: Obturator hernia presenting as small bowel obstruction. Am J Surg 1994; 167:396-398.

5. Yip AWC, Ah Chong AK, Lam KH: Obturator hernia: a continuing diagnostic challenge. Surgery 1993; 113: 266-269.

6. Yokoyama T, Munakata Y, Ogiwara M, et al: Preoperative diagnosis of strangulated obturator hernia using ultrasonography. Am J Surg 1997; 174: 76- 78. 


\section{CASE REPORT}

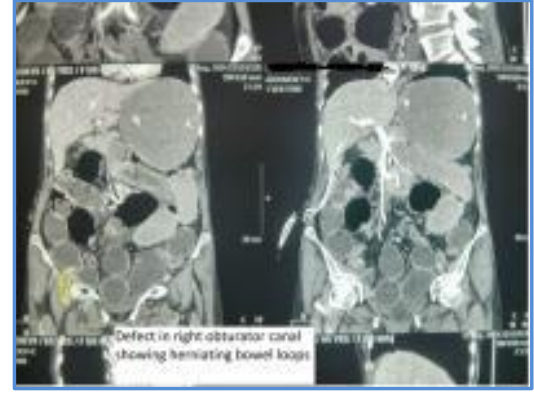

Fig. 1: C T Scan showing right sided Obturator Hernia

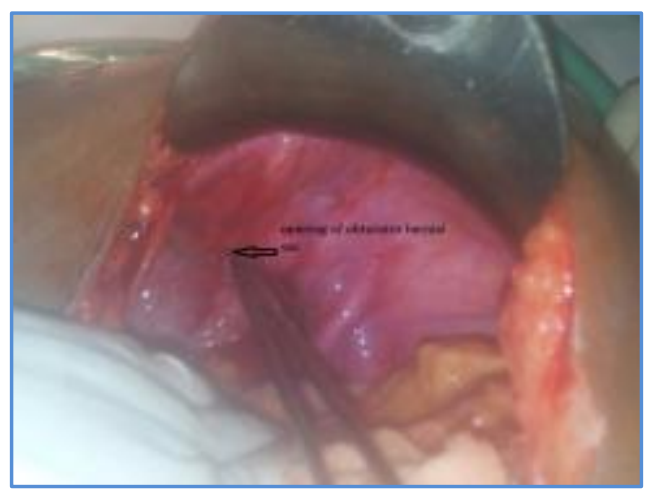

Fig. 3: Intra-operative photo showing opening of right sided obturator hernia

\section{AUTHORS:}

1. Minakshi Gadhire

2. Kanchan Sachanandani

3. Prashant Kadam

4. Mohan Joshi

\section{PARTICULARS OF CONTRIBUTORS:}

1. Associate Professor, Department of General Surgery, Lokmanya Tilak Medical College and Hospital, Mumbai.

2. Registrar, Department of General Surgery, Lokmanya Tilak Medical College and Hospital, Mumbai.

3. Assistant Professor, Department of General Surgery, Lokmanya Tilak Medical College and Hospital, Mumbai.

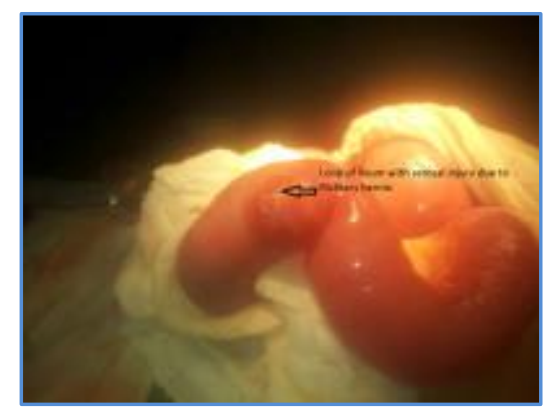

Fig. 2: Intra-Operative photo showing Ritchers type of hernia involving small bowel

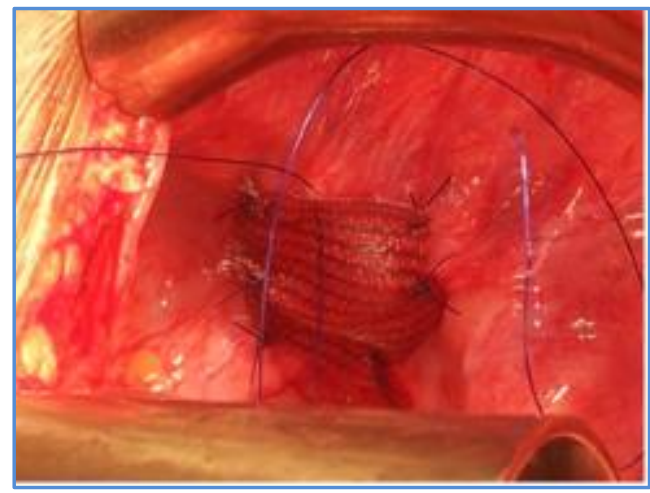

Fig. 4: Image showing meshplasty by sepramesh over the defect of right obturator hernia

4. Professor, Department of General Surgery, Lokmanya Tilak Medical College and Hospital, Mumbai.

\section{NAME ADDRESS EMAIL ID OF THE CORRESPONDING AUTHOR:}

Dr. Minakshi Gadhire,

Room No-25, Dept. of Surgery

First Floor, College Building,

Lokmanya Tilak Municipal Medical College \&

Hospital, Sion, Mumbai- 83.

E-mail: gadhireminakshi@yahoo.in

Date of Submission: 11/12/2014.

Date of Peer Review: 12/12/2014.

Date of Acceptance: 19/12/2014.

Date of Publishing: 29/12/2014. 